\title{
Incidence and Nutritional Management of Chylothorax after Surgery for Congenital Heart Diseases in Children
}

\author{
Jie Zheng, MS,${ }^{1}$ Ying-Yi Chen, BS, ${ }^{1}$ Chun-Ying Zhang, $\mathrm{MS}^{2}$, Wen-Qian Zhang, BS, ${ }^{1}$ Zhi-Yong Rao, $\mathrm{MD}^{1}$ \\ ${ }^{1}$ Department of Clinical Nutrition, West China Hospital of Sichuan University, Chengdu, Sichuan, China; \\ ${ }^{2}$ Department of Laboratory Medicine, West China Hospital of Sichuan University, Chengdu, Sichuan, China
}

\section{ABSTRACT}

Background: Chylothorax is caused by thoracic lymphatic system injury that leads to lymph extravasates in the thoracic cavity. Cardiac surgery was the most common cause. Reports comparing therapeutic effects between enteral nutrition (EN) with medium-chain triglycerides (MCT) and total parenteral nutrition (TPN) are few and inconsistent. Our study aimed to analyze the incidence of chylothorax in children in our hospital and optimum nutritional management modalities.

Methods: We retrospectively reviewed the medical records of children admitted to our hospital with a diagnosis of chylothorax from 2014 to 2018 . We analyzed the incidence of chylothorax, therapeutic effectiveness, and cost effectiveness of EN with MCT or TPN.

Results: 136 patients with chylothorax after surgery for congenital heart disease (CHD) were identified from 172 patients with chylothorax (79.07\%); chylothorax occurred in $5.62 \%$ of all 2420 congenital heart disease surgeries that were performed during that period. Tetralogy of Fallot (TOF), ventricular septal defect (VSD), and double-outlet right ventricle (DORV) were the most common primary diagnoses. Fontan surgery, TOF repair, and VSD repair were the most common primary procedures. We enrolled 45 patients with cured chylothorax. Nutrition support costs in the EN with MCT group $(\mathrm{n}=28)$ were significantly lower than in the TPN group $(\mathrm{n}=17)(P=.000)$. Time to resolution and time to removal of the drainage tube were shorter in EN with MCT versus TPN $(P=.003)$, and the length of hospital stay was shorter $(P=.032)$. There were no significant differences between the 2 groups in time from admission to surgery, postoperative days before diagnosing chylothorax, or length of PICU stay $(P>.05)$.

Conclusions: The therapeutic effects of EN with MCT were significantly better than those of TPN, with lower costs. Therefore, we suggest that EN with MCT be chosen first to treat chylothorax caused by surgery with mild chest drainage volume when gastrointestinal tract function is allowed.

Received fuly 23, 2020; received in revised form October 20, 2020; accepted October 21, 2020.

Correspondence: Zhi-Yong Rao, 37 Guoxue Lane, Wuhou District, Chengdu, Sichuan, China; 8618980602276 (e-mail: raoyong100@126.com)

\section{INTRODUCTION}

Chylothorax is a common complication with low incidence in children that can be congenital, traumatic, or due to high central venous pressure, malignancy, or other causes [SotoMartinez 2009]. Postoperative chylothorax is most common, and it occurs most frequently after surgery for congenital heart disease (CHD) [Mery 2014]. Chylothorax is associated with increased in-hospital morbidity [Mery 2014], prolonged hospitalization [Mery 2014; Haines 2014], and higher costs [Mery 2014].

No overall consensus or guidelines for chylothorax treatment are agreed on.4 Protocols and strategies differ according to physician experience and preference. Conservative treatment is generally the first line for chylothorax, and nutritional management is the main intervention. The mechanisms proposed for nutritional management are to reduce or inhibit long-chain fatty acid intake and absorption by the gastrointestinal tract, which can decrease the production of chyle. Nutrition management includes total parenteral nutrition (TPN), limiting dietary fat, and applying medium-chain triglycerides (MCT) into the diet or enteral nutrition supplement.

Resolution of chylothorax has been described with TPN (complete oral intake cessation) [Bond 1993], a fat-free/ low-fat diet [Chan 2010; Büttiker 1999], or an MCT plan [Chan 2010; Biewer 2010; Densupsoontorn 2005; Cormack 2004; Hermon 2019; Chan 2006] in children with variable success rates, but comparisons of the therapeutic effectiveness of nutrition support modalities in children are few and inconsistent. Nguyen et al [1995] reported that in 15 patients on TPN or a low-fat enteral diet, the average duration of lymph leak was 13.7 versus 13.2 days, respectively. Allen et al [1991] reported that an MCT-enriched diet (n $=12)$ and TPN $(\mathrm{n}=3)$ showed no difference in efficacy. Church et al [2017] reported that MCT diets showed higher success rates compared with TPN, but in their study, children with mild chest tube output were given enteral nutrition (EN), and time to resolution was not compared. We aimed to analyzed the incidence of chylothorax in children in our hospital and explore the optimal nutritional management method and cost-effectiveness of EN with MCT versus TPN. 


\section{METHODS}

Ethical approval for this study was obtained from the West China Hospital of Sichuan University Ethics Committee, approval number 2019 (896). Informed consent was waived by the West China Hospital of Sichuan University Ethics Committee. Trial registration: ChiCTR1900026916.

We retrospectively analyzed all patients (age 1 month to 18 years) diagnosed with chylothorax by a qualitative test of chyle (microscopic examination of the fluid stained with Sudan III showing fat globules) in West China Hospital of Sichuan University from 2014 to 2018. Clinical information was collected, including age, sex, gestational age, underlying diseases, procedures, nutrition management days and cost, duration and volume of chest tube drainage, and length of hospital and PICU stays.

\section{Trial Grouping and Nutrition Support Method}

Once chylothorax was confirmed, nutritional interventions were carried out. The daily energy and protein requirements for children referred to American Society of Parenteral and Enteral Nutrition/Academy of Nutrition and Dietetics and European Society of Parenteral and Enteral Nutrition relevant guidelines. All patients were treated initially with nothing per mouth (NPO), a low-fat diet, or TPN before 2016. After that, we gradually applied enteral nutrition with MCT formula based on recent research. Patients with high drainage output after surgery were treated with TPN initially and changed to EN with MCT or a low-fat diet when drainage decreased. Mild cases were treated with EN with MCT initially. However, nutritional management was still partly according to the physician's discretion and the patient's situation.

\section{EN with MCT Group}

Patients diagnosed with chylothorax treated by EN with MCT for $>3$ days were included in the EN with MCT group, including patients who were cured.

\section{TPN Group}

Patients diagnosed with chylothorax treated by TPN for $>3$ days were included in the TPN group, including patients who were cured. To balance comparability of the data, we chose patients admitted before 2016 for the TPN group.

Patients in the 2 groups with the following criteria were not included in the analysis: (1) EN with MCT changed to TPN; (2) nutrition support stopped for nontherapeutic reasons; (3) intake of foods outside the protocol; (4) the patient's condition worsened or the patient died; or 5) transfer to another institution.

\section{Statistical Analysis}

Statistics were analyzed with SPSS software, version 17.0. The normality of data was conducted using the Shapiro-Wilk test. Parametric data are expressed as mean \pm standard deviation and nonparametric data as median (minimum, maximum). The study used the 2-independent-samples test, $\chi^{2}$ test, and Fisher's test. The homogeneity of variance was tested before 2-independent-samples of parametric data. $P$ values $<.05$ were considered statistically significant.

\section{RESULTS}

\section{Basic Clinical Characteristics of Children Diagnosed with Chylothorax}

A total of 172 children were diagnosed with chylothorax from 2014 to 2018 in our hospital. The most common etiology was found to be surgery $(91.86 \%)$. Of the 172 patients, $140(81.40 \%)$ with chylothorax after heart surgery were identified, of whom $136(79.07 \%)$ underwent surgery for CHD (Table 1). Chylothorax occurred in $5.62 \%$ of all $2420 \mathrm{CHD}$ surgeries performed during that period.

\section{Data of Children after Surgery for CHD}

Primary diagnoses tetralogy of Fallot (TOF) $(\mathrm{n}=21)$, ventricular septal defect (VSD) $(\mathrm{n}=18)$, and double-outlet right ventricle $(\mathrm{DORV})(\mathrm{n}=13)$ ranked the top 3 in the 136 patients (males 89, females 47 ) after surgery for CHD. Fontan surgery $(\mathrm{n}=27)$, TOF repair $(\mathrm{n}=21)$, and VSD repair $(\mathrm{n}=18)$ were the top 3 primary operation types. Twenty-eight patients underwent second-time surgery, 21

Table 1. Diagnostic Categories of Children Diagnosed with Chylothorax $(\mathrm{N}=172)$

\begin{tabular}{lll}
\hline Diagnosis & Number & Rate (\%) \\
\hline Nonsurgery & & \\
Pleural effusion (undiagnosed cause) & 7 & 4.07 \\
Multiple serous effusion: nephrotic syndrome? & 2 & 1.16 \\
Tuberculosis? & 4 & 2.33 \\
Enlarged neck lymph nodes & 1 & 0.58 \\
Surgery & & \\
Congenital heart disease & 136 & 79.65 \\
Endocarditis & 2 & 1.16 \\
Pericarditis & 1 & 0.58 \\
Kawasaki disease & 1 & 0.58 \\
Diaphragmatic hernia & 5 & 2.91 \\
Intralobular lung sequestration & 1 & 0.58 \\
Congenital esophageal atresia & 1 & 0.58 \\
Traumatic esophageotracheal fistula & 1 & 0.58 \\
Liver transplantation (congenital liver fibrosis ) & 1 & 0.58 \\
Liver transplantation (biliary atresia ) & 3 & 1.74 \\
Scoliosis & 1 & 0.58 \\
Lymphangioma & 1 & 0.58 \\
Mediastinal mass & 1 & 0.58 \\
Huge space-occupying lesion of neck and chest & 1 & 0.58 \\
Thoracic giant tumor & 2 & 1.16 \\
& &
\end{tabular}


of whom had a Glenn procedure. Four patients had Ivemark syndrome and imperforate anus, and 3 patients had Down syndrome (Table 3).

\section{Basic Clinical Characteristics}

We analyzed 50 patients with chylothorax after surgery for congenital heart disease. They were divided into the EN with MCT group (32 patients) and the TPN group (18 patients). In the EN with MCT group, 3 patients changed to TPN because of unresolved chylothorax and 1 patient because of abdominal distention. In the TPN group, 1 patient had unresolved chylothorax and was discharged from hospital with a tube, but thoracic drainage volume was reduced. Of the 45 cured patients after surgery included in the analysis, 28 were treated by EN with MCT and 17 by TPN.

No significant differences between the EN with MCT group and the TPN group were found in sex, age, weight, or thoracic drainage volume at the time of chylothorax diagnosis $(P>.05)$ (Table 3$)$. TOF, pulmonary atresia $(\mathrm{PA})$, and VSD were the 3 most common diagnoses in both the EN with MCT group (8, 4, and 2, respectively) and the TPN group (3, 1 , and 2). Six patients underwent second-time surgery in both groups; 1 patient in each group had Ivemark syndrome, and 2 patients in the EN with MCT group and 1 in the TPN group had Down syndrome (Table 3).

\section{Comparisons of Clinical Outcomes with Different Nutrition Management Methods}

Forty-five cured patients were included in the analysis. The difference between the groups in total costs during hospitalization was not significant. Nutrition support costs, however, were significantly lower in the EN with MCT group than in the TPN group $(P=.000)$. Time to resolution and time to removal of the drainage tube were shorter in the EN with MCT group $(P=.003)$, as was the length of hospital stay $(P=.032)$. No significant differences were found between the groups in time from admission to surgery, postoperative days before diagnosing chylothorax, or length of pediatric intensive care unit (PICU) stay $(P>.05)$ (Table 4).

\section{DISCUSSION}

In children, the reported incidence of chylothorax after cardiac surgery is $0.85 \%$ to $9.2 \%$ [Soto-Martinez 2009; Haines 2014; Biewer 2010; Chan 2006; Purkait 2014; Milonakis 2009; Lin 2017; Mery 2014]. Cormack et al [2004] reported an incidence of $4.7 \%$, and Ismail et al [2014] reported 5\% (n $=57$ ), similar to our study incidence of $5.62 \%$ of all 2420 congenital heart disease surgeries. Haines et al [2014] reported that $65.1 \%$ of cases of chylothorax in children were associated with cardiac surgical procedures. In our study, this incidence was higher, at $79.07 \%(\mathrm{n}=136)$, but similar to reports by Church et al [2017] and Büttiker et al [1999], who reported incidences of $82 \%(\mathrm{n}=145)$ and $85 \%(\mathrm{n}=35)$, respectively. This range is likely due to the complexity of the procedures, the number of redo surgeries, and variations in age [Mery 2014; Buckley 2017].
Table 2. Data of Children after Surgery for CHD $(n=136)^{*}$

\begin{tabular}{|c|c|c|}
\hline Characteristic & Number & Rate (\%) \\
\hline $\operatorname{Sex}(M / F)$ & $89 / 47$ & \\
\hline Second-time surgery & 28 & 20.59 \\
\hline Glenn procedure & 21 & 15.44 \\
\hline Ivemark syndrome & 3 & 2.21 \\
\hline Imperforate anus & 1 & 0.74 \\
\hline Down syndrome & 3 & 2.21 \\
\hline \multicolumn{3}{|l|}{ Primary diagnosis } \\
\hline TOF & 21 & 16.18 \\
\hline VSD & 18 & 14.71 \\
\hline DORV & 13 & 9.56 \\
\hline PA & 11 & 8.09 \\
\hline Single ventricle & 10 & 7.35 \\
\hline Tricuspid atresia & 6 & 4.41 \\
\hline Coarctation of the aorta & 5 & 3.68 \\
\hline Transposition of great arteries & 4 & 2.94 \\
\hline \multicolumn{3}{|l|}{ Primary operation type } \\
\hline Fontan & 27 & 19.85 \\
\hline TOF repair & 21 & 15.44 \\
\hline VSD repair & 18 & 13.24 \\
\hline Glenn procedure & 9 & 6.62 \\
\hline DORV repair & 8 & 5.88 \\
\hline Correction of PA & 7 & 5.15 \\
\hline $\begin{array}{l}\text { Correction of total anomalous pulmonary } \\
\text { venous return }\end{array}$ & 4 & 2.94 \\
\hline Correction of coarctation of the aorta & 4 & 2.94 \\
\hline
\end{tabular}

*Primary diagnosis and primary operation are listed if $\mathrm{n}>3$. $\mathrm{COA}=$ Correction of Coarctation of the Aorta; $\mathrm{VSD}=$ ventricular septal defect; TOF = Totralogy of Fallot; TAPVR = Total anomalous pulmonary venous return; $\mathrm{SV}$ = single ventricle; $\mathrm{DORV}$, double outlet right ventricle; TGA, transposition of great arteries; PA = pulmonary atresia.

Ismail et al [2014] reported that single repair (GlennFontan) (27\%), aortic arch repairs (18\%), and VSD repairs (18\%) were the 2 most common surgeries complicated by chylothorax; according to Haines et al [2014], they were Fontan procedure and TOF repair; and according to Lin et al [2017], they were patent ductus arteriosus ligation and VSD repair. Similarly, in our study, TOF, VSD, and DORV were the most common primary diagnoses, and Fontan, TOF repair, and VSD repair the top 3 primary operation procedures. Procedures with high complexity and systemic venous hypertension, such as TOF repair and Fontan procedures, are reported as causing increased risk of chylothorax.

Resolution of chylothorax has been reported with variable success rates, but comparisons between EN with MCT and 
Table 3. Basic Clinical Characteristics of the 2 Groups $(n=45)^{*}$

\begin{tabular}{lcc}
\hline Characteristic & MCT $(\mathrm{n}=28)$ & TPN $(\mathrm{n}=17)$ \\
\hline Sex $(\mathrm{M} / \mathrm{F})$ & $18 / 10$ & $14 / 3$ \\
Age $(\mathrm{mo})$ & $32.00(1.00,129.00)$ & $35.00(6.00,128.00)$ \\
Weight $(\mathrm{kg})$ & $10.85(3.30,31.00)$ & $17.00(4.00,23.50)$ \\
First day drainage volume $(\mathrm{mL} / \mathrm{kg})$ & $11.43(0.58,80.34)$ & $13.50(2.11,95.35) \dagger$ \\
Primary diagnosis: TOF, PA, VSD, tricuspid atresia & $8 / 4 / 2 / 0$ & $3 / 1 / 2 / 2$ \\
Primary surgical type: TOF repair, Fontan, VSD repair, & $8 / 7 / 1 / 1$ & $4 / 5 / 2 / 2$ \\
Glenn & & 6 \\
Second-time surgery & 6 & .101 \\
Ivemark syndrome & 1 & 1 \\
Down syndrome & 2 & 1 \\
\hline
\end{tabular}

*Data are median (minimum, maximum) or $\mathrm{n}$.

$\lceil n=15: 2$ cases of first-day drainage volume were not recorded.

Table 4. Clinical Outcomes of the 2 Groups $(n=45)^{*}$

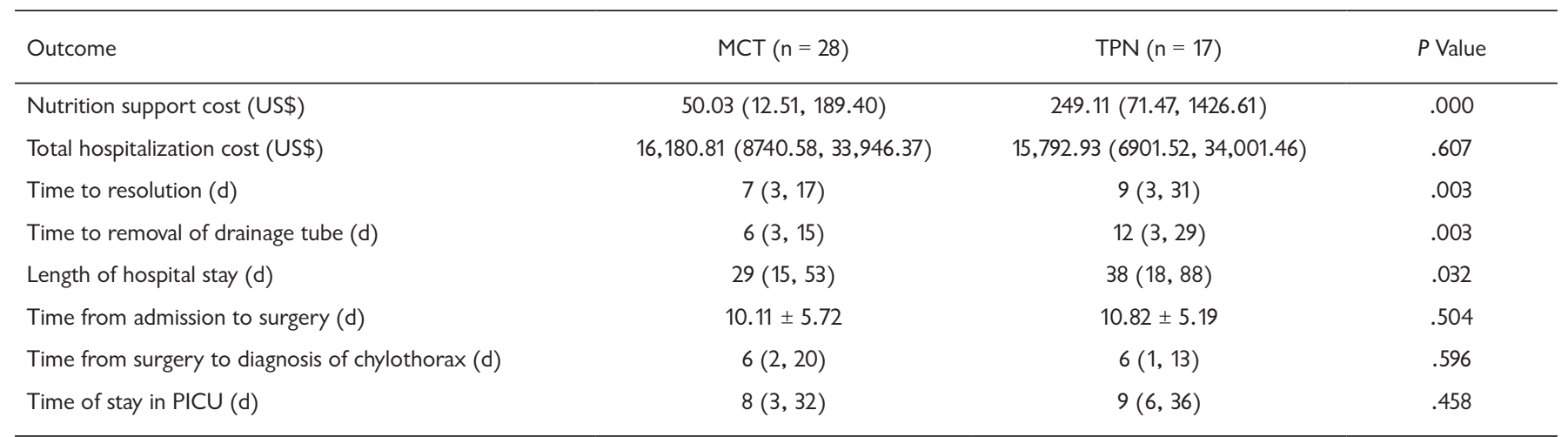

*Data are median (minimum, maximum) or mean \pm standard deviation.

TPN are few. Church et al [2017] reported that MCT diets showed higher success rates compared with TPN, but in their study, children with mild chest tube output were given EN. In our study, 28 patients receiving EN with MCT were cured out of 32 patients, and 17 patients receiving TPN were cured out of 18 patients, with similar drainage outputs on the first day of nutritional management $(P>.05)$.

We demonstrated a significant decrease in duration of chest tube utilization in children given EN with MCT. Time to resolution and time to removal of the drainage tube have been variable in different studies. Bond et al [1993] reported an average drainage duration of 11.9 days (range 4 to 30 ) with TPN management. Biewer et al [2010] reported that chylothorax in 17 newborns and infants who received an MCT diet was resolved successfully in a median of 9 days. Allen et al [1991] reported that the average time on a drainage tube was $11.3 \pm 8.4$ days in the MCT-enriched diet group $(n=12)$ and $9.5 \pm 6.4$ days in the TPN group $(\mathrm{n}=3)$. In our study, time to resolution was a median of 7 days (range 3 to 17 ) in the EN with MCT group and 9 days (range 3 to 31 ) in the TPN group, and time to removal of drainage tube was a median of 6 days (range 3 to 15) and 12 days (range 3 to 29), respectively. We noticed that in the EN with MCT group, the median time to resolution (time to finish nutritional management) was similar to the time to removal of the drainage tube. In the TPN group, the drainage tube was removed later than resolution of chylothorax, which might be considered a conservative observation of recurrence.

The nutrition support costs in the EN with MCT group were lower than in the TPN group $(P=.000)$, but the difference in total costs during hospitalization was not significant. Chylothorax has been diagnosed a median of 4 to 9 days after surgery [Chan 2010; Chan 2006; Winder 2018; Yeh 2013]; in our study, it 6 days in both groups. The length of PICU stay was a median of 8 days in EN with 
MCT and 9 days in TPN $(P>.05)$, which was similar to reports by Yeh et al [2013] and Winder et al [2018]. The length of hospital stay was significantly shorter in the EN with MCT group, partly because of the shorter time of chest tube utilization.

\section{Limitations}

Limitations of this study include the following: our sample size was from a single institution; our study was retrospective; some relevant information, such as the daily chest drainage output, was recorded only partially; and the diagnosis of chylothorax used a qualitative test, which may result in lower sensitivity.

\section{Conclusion}

In our study, chylothorax occurred in $5.62 \%$ of all 2420 congenital heart disease surgeries. Fontan surgery, TOF repair, and VSD repair were the most common primary procedures. The therapeutic effects of EN with MCT were significantly better than those of TPN, with lower costs, and enteral nutrition is associated with fewer complications. Therefore, we suggest that EN with MCT be chosen first to treat chylothorax caused by surgery with mild chest drainage volume when gastrointestinal tract function is allowed. We also suggest that consensus and guidelines are needed to achieve uniformity and optimize management modalities for chylothorax.

\section{ACKNOWLEDGMENTS}

This research was funded by the Department of Science and Technology of Sichuan Province (grant 2019YJ0042 to Z-Y.R.).

\section{REFERENCES}

Allen EM, van Heeckeren DW, Spector ML, Blumer JL. Management of nutritional and infectious complications of postoperative chylothorax in children. J Pediatr Surg 1991;26:1169-1174.

Biewer ES, Zurn C, Arnold R, et al. Chylothorax after surgery on congenital heart disease in newborns and infants-Risk factors and efficacy of MCT-diet. J Cardiothorac Surg 2010;5:127.

Bond SJ, Guzzetta PC, Snyder ML, Randolph JG. Management of pediatric postoperative chylothorax. Ann Thorac Surg 1993;56:469-472.

Buckley JR, Graham EM, Gaies M, et al. Clinical epidemiology and centre variation in chylothorax rates after cardiac surgery in children: A report from the Pediatric Cardiac Critical Care Consortium. Cardiol Young 2017:1-8.
Büttiker V, Fanconi S, Burger R. Chylothorax in children: Guidelines for diagnosis and management. Chest 1999;116:682-687.

Chan EH, Jennifer LR, William GW, Glen SVA, John GC, Brian WM. Postoperative chylothorax after cardiothoracic surgery in children. 2010;80:1864-1870.

Chan SY, Wendy L, Wilfred HSW, Lik-cheung C, Adolphus KTC, Yiufai C. Chylothorax in children after congenital heart surgery. Ann Thorac Surg 2006;82:1650-1657.

Church JT, Antunez AG, Dean A, et al. Evidence-based management of chylothorax in infants. J Pediatr Surg 2017:S0022346817301598.

Cormack BE, Wilson NJ, Finucane K, West TM. Use of Monogen for pediatric postoperative chylothorax. Ann Thorac Surg 2004;77:301-305.

Densupsoontorn NS, Jirapinyo P, Wongarn R, et al. Management of chylothorax and chylopericardium in pediatric patients: Experiences at Siriraj Hospital, Bangkok. Asia Pac J Clin Nutr 2005;14:182-187.

Haines C, Walsh B, Fletcher M, Davis PJ. Chylothorax development in infants and children in the UK. Arch Dis Childhood 2014;99:724-730.

Hermon M, Tenner E, Burda G, Strohmaier W, Schlager G, Golej J. Chylothorax and chylous-like diseases in children: Clinical management. Front Pediatr 2019;7:258.

Ismail SR, Kabbani MS, Najm HK, Shaath GA, Jijeh AMZ, Hijazi OM. Impact of chylothorax on the early post operative outcome after pediatric cardiovascular surgery. J Saudi Heart Assoc 2014;26:87-92.

Lin C-H, Lin W-C, Chang J-S. Presentations and management of different causes of chylothorax in children: one medical center's experience. Biomedicine (Taipei) 2017;7:5.

Mery CM, Moffett BS, Khan MS, et al. Incidence and treatment of chylothorax after cardiac surgery in children: analysis of a large multi-institution database. J Thorac Cardiovasc Surg 2014;147:678-686.

Milonakis M, Chatzis AC, Giannopoulos NM, et al. Etiology and management of chylothorax following pediatric heart surgery. J Card Surg 2009;24:369-373.

Nguyen DM, Shum-Tim D, Dobell ARC, Tchervenkov CI. The management of chylothorax/chylopericardium following pediatric cardiac surgery: A 10-year experience. J Cardiac Surg 1995;10:302-308.

Purkait R, Saha A, Tripathy I, Roy B. Congenital chylous ascites treated successfully with MCT-Based formula and octreotide. J Indian Assoc Pediatr Surg 2014;19:175-177.

Soto-Martinez M, Massie J. Chylothorax: Diagnosis and management in children. Paediatr Respir Rev 2009;10:199-207.

Winder MM, Eckhauser AW, Delgado-Corcoran C, Smout RJ, Marietta J, Bailly DK. A protocol to decrease postoperative chylous effusion duration in children. Cardiol Young 2018;28:816-825.

Yeh J, Brown ER, Kellogg KA, et al. Utility of a clinical practice guideline in treatment of chylothorax in the postoperative congenital heart patient. Ann Thorac Surg 2013;96:930-936. 